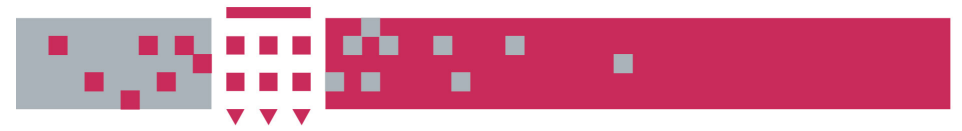

WestminsterResearch

http://www.wmin.ac.uk/westminsterresearch

\title{
On the structure and performance of a novel blind source separation based carrier phase synchronization error compensator.
}

\author{
Ediz Cetin \\ Izzet Kale \\ Richard Morling
}

Cavendish School of Computer Science

Copyright (C [2002] IEEE. Reprinted from 45th Midwest Symposium on Circuits and Systems (MWSCAS-2002), 04-07 Aug 2002, Tulsa, USA.

This material is posted here with permission of the IEEE. Such permission of the IEEE does not in any way imply IEEE endorsement of any of the University of Westminster's products or services. Internal or personal use of this material is permitted. However, permission to reprint/republish this material for advertising or promotional purposes or for creating new collective works for resale or redistribution must be obtained from the IEEE by writing to pubs-permissions@ieee.org. By choosing to view this document, you agree to all provisions of the copyright laws protecting it.

The WestminsterResearch online digital archive at the University of Westminster aims to make the research output of the University available to a wider audience. Copyright and Moral Rights remain with the authors and/or copyright owners. Users are permitted to download and/or print one copy for non-commercial private study or research. Further distribution and any use of material from within this archive for profit-making enterprises or for commercial gain is strictly forbidden.

Whilst further distribution of specific materials from within this archive is forbidden, you may freely distribute the URL of WestminsterResearch. (http://www.wmin.ac.uk/westminsterresearch).

In case of abuse or copyright appearing without permission e-mail wattsn@wmin.ac.uk. 


\title{
On the Structure and Performance of a Novel Blind Source Separation Based Carrier Phase Synchronization Error Compensator
}

\author{
Ediz Çetin, Izzet Kale and Richard C. S. Morling \\ University of Westminster, Department of Electronic Systems, \\ Applied DSP and VLSI Research Group, \\ London W1W 6UW, United Kingdom
}

\begin{abstract}
In this paper we carry out a detailed performance analysis of a novel blind-source-separation (BSS) based DSP algorithm that tackles the carrier phase synchronization error problem. The results indicate that the mismatch can be effectively compensated during the normal operation as well as in the rapidly changing environments. Since the compensation is carried out before any modulation specific processing, the proposed method works with all standard modulation formats and lends itself to efficient real-time custom integrated hardware or software implementations.
\end{abstract}

\section{INTRODUCTION}

With the growing demand for multiband/ multimode wireless devices that can handle high-speed data transmission over band-limited channels, there is a need for cost-effective receiver solutions, which circumvent the overhead associated with traditional approaches. The large signal constellations of $M$-QAM/M-PSK impose stringent constraints on the quality of the carrier acquisition algorithms to be used [1]. This coupled with the high data rates at which these systems operate, implies that not only the algorithm must perform well, but also it must at the same time be simple to implement.

Several acquisition and tracking methods have been reported in the literature tackling the carrier phase synchronization problem [2]-[6]. The majority of these methods rely on non-linear operation, e.g. cubic nonlinearity to function properly. This is needed to remove the modulation dependent phase shifts. Initially the phase error is estimated and then corrected. This paper explores the structure and the performance capability of a non-data aided feed-forward adaptive DSP technique developed for quadrature receivers in [7].

The paper is organized as follows: Section 2 defines the model of the carrier phase synchronization errors and the compensator structure. Section 3 describes the performance analysis and the simulation results, while concluding remarks are given in Section 4.

\section{SYSTEM DESCRIPTION}

\subsection{BSS based Carrier Phase Synchronization Error Correction:}

In the theoretical derivation of the algorithm the following notations will be used:

$$
\begin{aligned}
\text { Transmitted I/Q Signals: } & \mathbf{s}(k)=\left[\begin{array}{ll}
s_{I}(k) & s_{Q}(k)
\end{array}\right]^{T} \\
\text { Received I/Q Signals: } & \mathbf{r}(k)=\left[\begin{array}{ll}
r_{l}(k) & r_{Q}(k)
\end{array}\right]^{T}
\end{aligned}
$$

In the presence of carrier phase synchronization errors the erroneous downconverted baseband signal $\mathbf{r}(k)$ can be expressed as [7]:

$$
\mathbf{r}(k)=\mathbf{H s}(k)
$$

where $\mathbf{H}$ is the unknown nonsingular mixing matrix which is determined by the carrier phase error and $\mathbf{s}(k)$ is the transmitted signal [7]. Recasting (1) in a 2-by-2-matrix form we get [7]:

$$
\left[\begin{array}{l}
r_{I}(k) \\
r_{Q}(k)
\end{array}\right]=\underbrace{\left[\begin{array}{cc}
\cos \phi & -\sin \phi \\
\sin \phi & \cos \phi
\end{array}\right]}_{H} \times\left[\begin{array}{l}
s_{J}(k) \\
s_{Q}(k)
\end{array}\right]
$$

where $\phi=\hat{\theta}-\theta$, is the time varying phase error in radians between the transmitter's phase, $\theta$ and the receiver's phase estimate $\hat{\theta}$. As it can be seen from (2), due to the carrier phase synchronization errors the quadrature component is not only attenuated by $\cos \phi$, but also there is crosstalk between them. Hence, they are no longer uncorrelated and orthogonal. Since the average power levels of $s_{1}(k)$ and $s_{\mathrm{Q}}(k)$ are similar, a small phase error causes a large degradation in performance [1].

Given the received vector $\mathbf{r}(k)$, the source separation problem consists of recovering the original signals in an unsupervised way by finding a de-mixing matrix $\mathbf{W}$ hence recovering the sources:

$$
\begin{aligned}
\mathbf{c}(k) & =\mathbf{W r}(k) \\
& =\mathbf{W H s}(k) \\
& \approx \mathbf{s}(k)
\end{aligned}
$$


The application of the BSS to Carrier Phase Synchronization error correction is depicted in Figure 1.

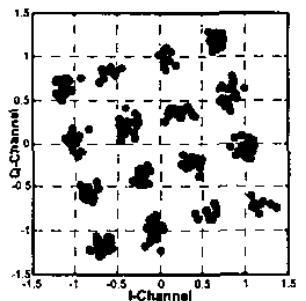

Received
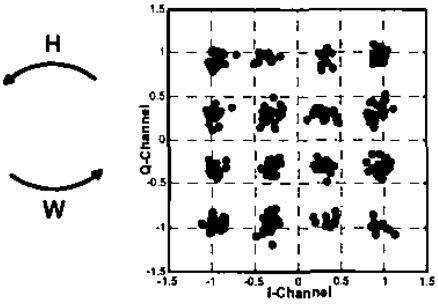

Transmitted
Figure 1. Application of BSS to Carrier Phase Synchronization Error Correction.

As it can be seen from Fig. 1, the mixing matrix $\mathbf{H}$ transforms the transmitted $s_{\mathrm{I}}(k)$ and $s_{\mathrm{Q}}(k)$ signals to new received $r_{\mathrm{i}}(k)$ and $r_{\mathrm{Q}}(k)$ signals. As $\phi$ grows larger, the projection of a particular constellation point rotates in the signal space and gets closer to the edge of its decision region. As a result, it takes less noise power to perturb the projection and move it into wrong decision region. The result is a higher probability of bit-error-rate than would otherwise be expected. The resulting loss in performance is obviously undesirable. The de-mixing matrix $\mathbf{W}$ on the other hand transforms the received $r_{\mathrm{l}}(k)$ and $r_{\mathrm{Q}}(k)$ signals back to the original transmitted ones, eliminating the effects of imperfect carrier phase synchronization.

\subsection{Structures for the Solution}

In the theoretical derivation of the algorithm the following notations will be used:

$$
\begin{aligned}
& \text { Transmitted I/Q Signals: } \quad \mathbf{s}(z)=\left[\begin{array}{ll}
s_{I}(z) & s_{Q}(z)
\end{array}\right]^{T} \\
& \text { Received I/Q Signals: } \quad \mathbf{r}(z)=\left[r_{I}(z) r_{Q}(z)\right]^{T} \\
& \text { Corrected I/Q Signals: } \quad \mathbf{c}(z)=\left[\begin{array}{ll}
c_{I}(z) & c_{Q}(z)
\end{array}\right]^{T} \\
& \text { Mixing Vector: } \quad \mathbf{H}_{i}^{(k)}(z)=\left[h_{i}^{(k)}(0) \ldots h_{i}^{(k)}\left(L_{i}\right)\right]^{T} \\
& \text { Coefficient Vector: } \quad \mathbf{W}_{i}^{(k)}(z)=\left[w_{i}^{(k)}(0) \ldots w_{i}^{(k)}\left(L_{i}\right)\right]^{T}
\end{aligned}
$$

where $L_{i}$ is the filter order and $i=1,2$. In this section we will first derive the general properties of the solution to the carrier phase synchronization error correction before discussing any specific criterion or adaptive method. The only assumption we make is that the transmitted signals, $s_{1}(k)$ and $s_{\mathrm{Q}}(k)$ are orthogonal and not correlated witch each other. Hence, this assumption implies that:

$$
E\left[r_{l}(k) \times r_{Q}(k-n)\right]=0, \quad \forall n,
$$

where $E[\bullet]$ denotes expectation. The mixing matrix can be further simplified as:

$$
\mathbf{H}=\left[\begin{array}{cc}
1 & -\sin \phi \\
\sin \phi & 1
\end{array}\right] \equiv\left[\begin{array}{cc}
1 & H_{2}(z) \\
H_{1}(z) & 1
\end{array}\right]
$$

A possible feed-forward solution to the source separation problem is depicted in Fig. 2.

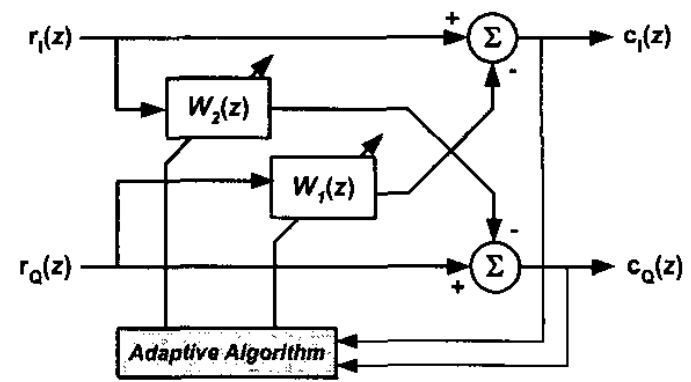

Figure 2. BSS-based I/Q corrector [7]

The source estimates, $\mathrm{c}_{\mathrm{I}}(z)$ and $\mathrm{c}_{\mathrm{Q}}(z)$, can be expressed as:

$$
\begin{aligned}
& c_{l}(z)=\left(1-W_{1}(z) H_{2}(z)\right) s_{l}(z)+\left(H_{1}(z)-W_{1}(z)\right) s_{Q}(z) \\
& c_{Q}(z)=\left(H_{2}(z)-W_{2}(z)\right) s_{l}(z)+\left(1-W_{2}(z) H_{1}(z)\right) s_{Q}(z)
\end{aligned}
$$

When the filters converge, i.e. $W_{1}(z)=H_{1}(z)$ and $W_{2}(z)=H_{2}(z)$ then the source estimates become:

$$
\begin{aligned}
& c_{I}(z)=\left(1-H_{1}(z) H_{2}(z)\right) s_{I}(z) \\
& c_{Q}(z)=\left(1-H_{1}(z) H_{2}(z)\right) s_{Q}(z)
\end{aligned}
$$

As it can be seen from (7) the I and Q channels have the same gain and are once again orthogonal. Also, $\left(1-H_{1}(z) H_{2}(z)\right) \approx 1$ and can be safely ignored. An alternative implementation for the separation structure can be found by placing the filters in the feedback loop. The FIR filters $W_{1}(z)$ and $W_{2}(z)$ are adapted using the simple LMS algorithm [8].

\section{SIMULATION RESULTS}

To analyze the performance of the proposed structure, we consider linearly modulated communications signals, namely $M$-PSK and $M$-QAM with ideal symbol rate sampling. We assume an AWGN channel with SNR of 20 $\mathrm{dB}$ and carrier phase synchronization error of $15^{\circ}$. The performance of the adaptive algorithm is characterized by the modeling-error. The modeling error is defined as the squared norm of the difference of the transfer functions between the original mixing filters and the estimated filters, relative to the squared norm of the mixing filter. It is given as:

$$
M E R R_{w_{i}}=\frac{\left|H_{i}(z)-W_{i}(z)\right|^{2}}{\left|H_{i}(z)\right|^{2}} \quad i=1,2
$$

The time domain modeling error is defined as the expected value of the sum of squares of the difference between the original and the estimated filters. It is expressed as follows: 


$$
\varepsilon_{i}(k)=\frac{E\left[\sum_{i=0}^{L i}\left(h_{i}[l]-w_{i}^{k}[l]\right)^{2}\right]}{\sum_{l=0}^{L i} h_{i}^{2}[l]}
$$

\subsection{Influence of Filter Order}

Modeling errors for different filter orders $(L)$ using 16QAM modulated signals with $15^{\circ}$ synchronization error is shown in Fig. 3.

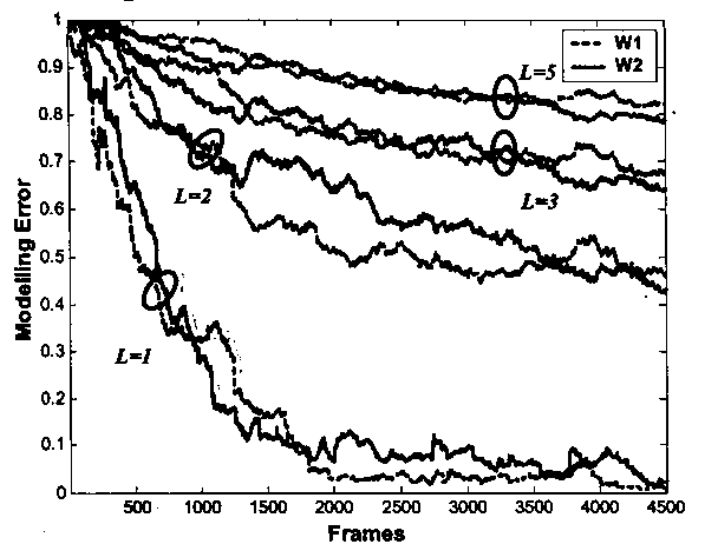

Figure 3. Modeling Error for different filter orders $(L)$

From Fig. 3 we can see that longer filters converge slower. What is more, increase of the filter tap lengths leads to larger misadjustment as expected [8]. Hence, filter order of 1 (i.e. $l_{i}=2$-taps) is chosen for the proposed algorithm.

\subsection{Influence of Step-size $(\mu)$}

Modeling error for different step-sizes $(\mu)$ using 16-QAM modulated signals with $15^{\circ}$ synchronization error is shown in Fig. 4.

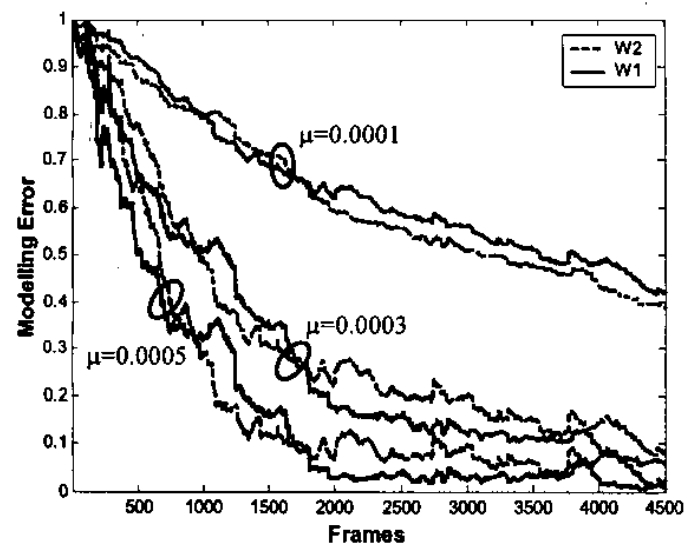

Figure 4. Modeling Error for different step-sizes $(\mu)$

Step-size values smaller than 0.0005 and larger than 0.001 , that made the system unstable were discarded from the Fig. 4. As it can be seen from Fig. 4 step-size, $\mu=0.0005$, gives the best performance.

\subsection{Time Varying Environments}

We will now examine the effect of time-varying imbalances on the adaptation and on the corresponding compensation performance. For the first 5500 frames, the carrier phase error is $15^{\circ}$. After that, an abrupt change from $15^{\circ}$ to $7.5^{\circ}$ is made at frame 5500 . After 4500 frames the carrier phase error is changed linearly from $7.5^{\circ}$ to reach $9^{\circ}$ for the next 1000 frames. The results are illustrated in Fig. 5.

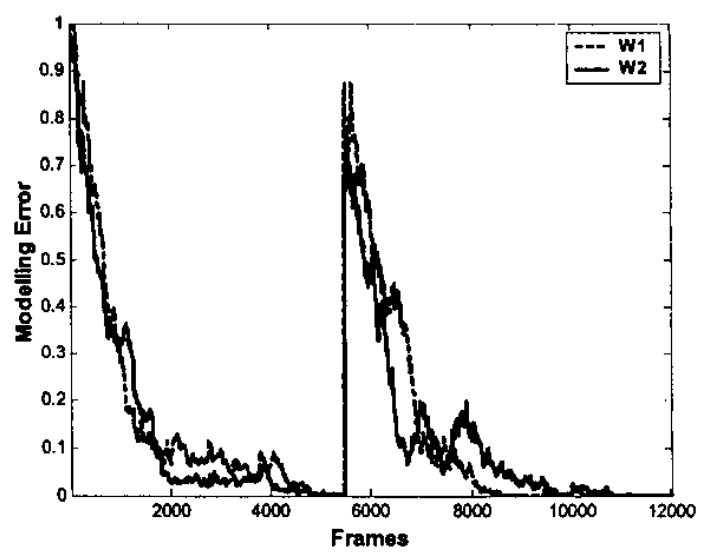

Figure 5. Tracking performance

As it can be seen from Fig. 5, a sudden change in the mixing coefficients does not cause the algorithm to diverge and the filters track the changes rapidly and the modeling error is zeroed. In addition, a time-variant mixture does not affect the compensation performance. This indicates that the proposed method is also capable of tracking time-varying synchronization errors.

\subsection{Different Modulation Formats}

Figs. 6 to 8 depict the constellation and eye diagrams for the application of the BSS-based corrector to 8-PSK, and 16-QAM modulation formats with AWGN Channel, SNR of $20 \mathrm{~dB}, L=1$ and $\mu=0.0005$ respectively.

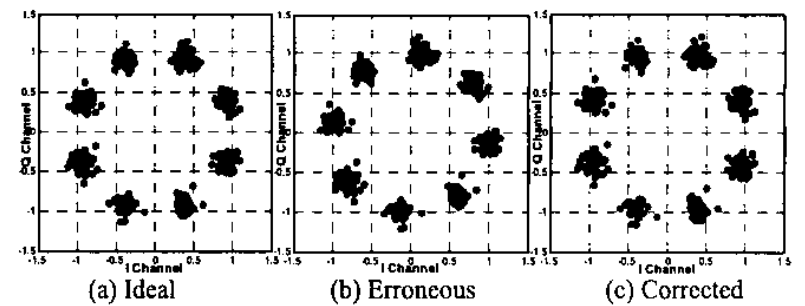

Figure 6. Constellation diagrams for (a) Ideal, (b) Erroneous and (c) Corrected 


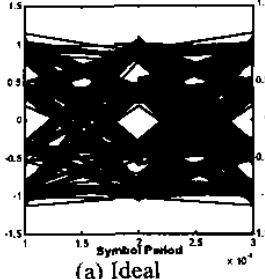

(a) Ideal

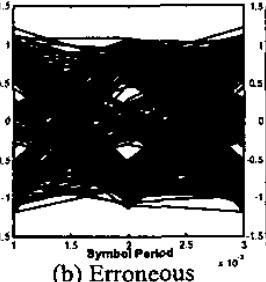

(b) Erroneous

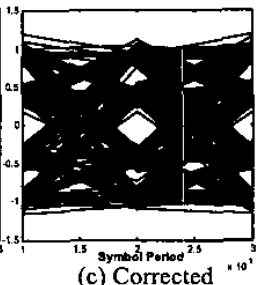

(c) Corrected
Figure 7. Eye diagrams for (a) Ideal, (b) Erroneous and (c) Corrected

As it can be seen from the constellation diagrams of Fig. 6 , the output of our adaptive algorithm (c) matches the original (a). The erroneous constellation diagram rotated by $15^{\circ}$ (b) is de-rotated back to the original. Fig. 7 depicts the eye diagrams for the ideal (a), erroneous (b) and corrected (c) system outputs. As it can be seen from (b) the eye diagram of the erroneous system is somewhat closed. The output of our adaptive system as shown in Fig. 7 (c) substantially opens the eye converting it back to the original state as shown in (a).

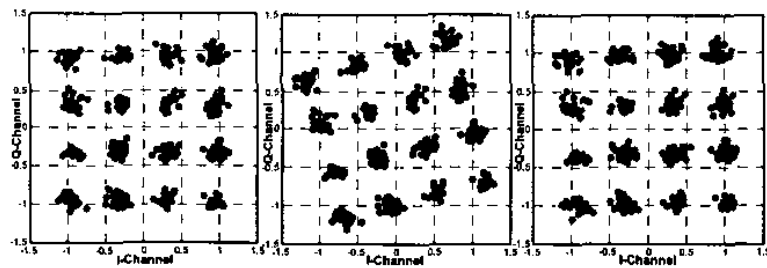

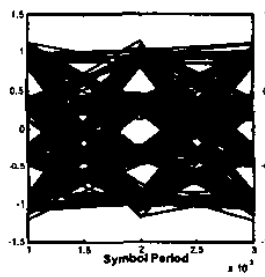

(a) Ideal

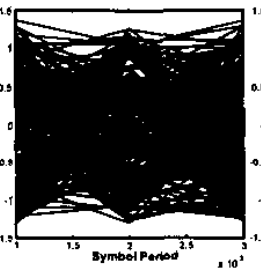

(b) Erroneous

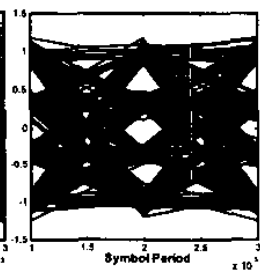

(c) Corrected
Figure 8. Constellation and Eye diagrams for (a) Ideal, (b) Erroneous and (c) Corrected

Once again observing the constellation diagrams of Fig. 8, the output of our adaptive algorithm (c) matches the original (a). The erroneous constellation diagram rotated by $15^{\circ}$ (b) is de-rotated back to the original. In addition to this, once again the output of our adaptive system as shown in Fig. 8 (c) substantially opened the closed eye diagram of the erroneous system (b) converting it back close to the original state as shown in (a).

Modeling error for 8-PSK and 16-QAM cases are depicted in Fig. 9. As it can be seen, the de-mixing filters $W_{1}$ and $\mathrm{W}_{2}$ almost match the mixing filters $\mathrm{H}_{1}$ and $\mathrm{H}_{2}$; hence the modeling errors are almost zeroed. Therefore, the effects of the imperfect carrier phase synchronization are eliminated. Resulting in lower bit-error-rates than would otherwise be achieved if no compensation was carried out.

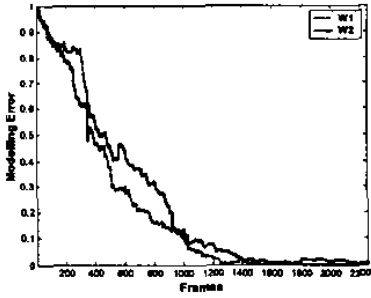

(a)

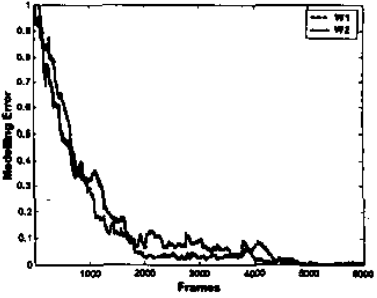

(b)
Figure 9. Modeling Error for (a) 8-PSK (b) 16-QAM

\section{CONCLUDING REMARKS}

In this paper we have presented the analytical results for the performance of a simple, low-complexity, modulation format independent, BSS based compensator to combat the carrier phase synchronization problem in wideband digital receivers. The robustness of the algorithm in terms of step-size, filter-tap lengths and against different modulation formats; $M$-QAM and $M$-PSK and constellation sizes were demonstrated through simulations. What is more, the algorithms ability to function properly in rapidly changing environments and time varying phase synchronization errors has also been demonstrated The presented results demonstrate substantial improvements indicating that the BSS structure can offer adequate performance for most communication systems

\section{REFERENCES}

[1] John G. Proakis, "Digital Communications, 4th Edition", McGrawHill, 2001, ISBN: 0-07-232122-3.

[2] Classen, F.; Meyr, H.; Sehier, P., "An all feedforward synchronization unit for digital radio", 43rd IEEE Vehicular Technology Conference, pp. $738-741,1993$

[3] Moeneclaey and $M$. de Jonghe G., "ML-oriented NDA carrier synchronization for general rotationally symmetric signal constellations", IEEE Transactions on Communications, vol. 42 Issue: 8, pp. $2531-2533$, August 1994

[4] Kil Nam Oh and $Y$ O Chin, "Modified Constant Modulus Algorithm: Blind Equalization and Carrier Phase Recovery Algorithm" Proceedings of IEEE International Conference on Communications (ICC '95), Seattle, vol: 1, pp. $498-502$, 1995

[5] Ali Abu-Rgheff, M.N.; Sumartana, I.G.G., "Carrier phase tracking in digital radio communications" Electronics Letters Volume: 34 24, pp. $2306-2307,26$ Nov. 1998.

[6] Morlet, C.; Boucheret, M.-L.; Buret, I., “ Low-Complexity CarrierPhase Estimator Suited to On-Board Implementation" IEEE Transactions on Communications, vol. 489 , ppl451-1454, Sept. 2000.

[7] Cetin, E., I. Kale and R. C. S. Morling, "Blind Source Separation Based Low-Complexity Carrier Phase Synchronization", International Symposium on Signal Processing and Information Technology (ISSPIT 2001), Cairo, Egypt, 28-30 December 2001.

[8] Widrow B. and S.D. Stearns, "Adaptive Signal Processing", Prentice Hall, 1985 ISBN: 0-13-004029-0 